THP-32(T5)

Multiphoton characterization of tissue engineering scaffolds Tzu-Yu Lin1, Sung-Jan Lin2, Hsiao-Ching Chen3, Hsuan-Shu Lee3, Shu-Wen Teng1, Wen Lo1, Chen-Yuan Dong1*

1:Department of Physics, Nation Taiwan University

2:Department of Dernmatology, National Taiwan University Hospital

3:Department of Internal Medicine, National Taiwan University Hospital and College of

Medicine

*To whom correspondence should be addressed: cydong@phys.ntu.edu.tw

Tel.: 886-2-3366-5155 ; Fax : 886-2-3366-5244

\begin{abstract}
The use of seeding scaffolds has facilitated advances in tissue engineering. We found that multiphoton microscopy is useful in imaging commonly used tissue engineering scaffolds such as polyglycolic acid (PGA).

Introduction

The use of seeding scaffolds has facilitated advances in tissue engineering. While traditional imaging modalities are useful in revealing structural changes to engineered tissues, it is difficult to follow dynamic events tissues in these structures. In this work, we investigate the possibilities of using multiphoton microscopy, a minimally invasive bioimaging technique, by characterizing scaffolds in tissue engineering applications. We found that multiphoton microscopy is useful in imaging commonly used tissue engineering scaffolds such as polyglycolic acid (PGA).
\end{abstract}

We hope to use multiphoton microscopy to obtain multiphoton signatures of tissue engineering scaffolds and to use such information in tissue engineering applications.

The schematic of the multiphoton microscope used in this study is shown in Fig. 1.

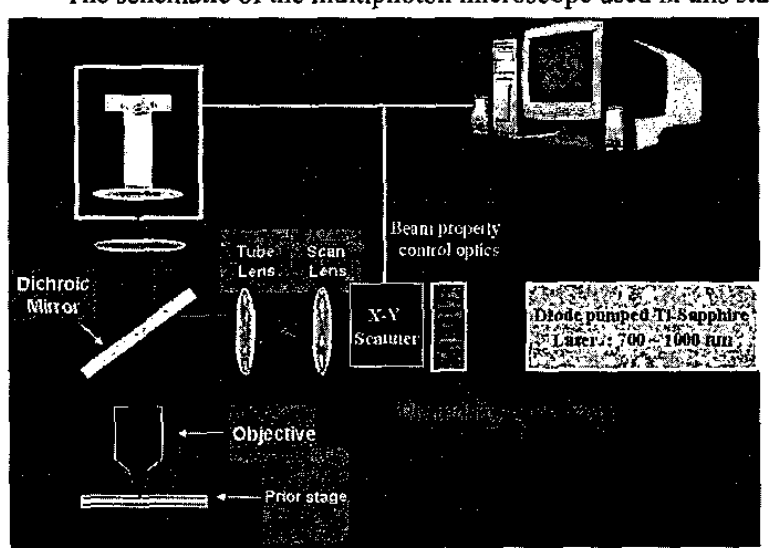

Fig1. Two-photon microscopy setup

\title{
Motivation and goal
}

The extracelluar matrix play important roles in organisms. It provides an adhesive substrate that serves as a 3D physical support matrix. It also promotes signaling pathways which influence cell migration, proliferation, and differentiation. For example, collagen fibers of extracelluar matrix serves the function of supporting skins. While it is difficult to simulate the exact environment of tissue growth, various scaffold materials have been used to in tissue engineering applications as substrates for tissue growths. Some common features of many of the scaffolds are their ability of being biocompatible, biodegradable, and porous.

\section{Result and Discussion}




\section{THP-32(T5)}

Shown in Fig. 2 is the multiphoton image of polyglycolic acid (PGA) scaffold (Synthecon). The image shows that the PGA scaffold provides a strong multiphoton signature. The fiber alignment is clearly visible.

And, shown in Fig.3 is the multiphoton image of lactic acid (PLA) scaffold (Synthecon).

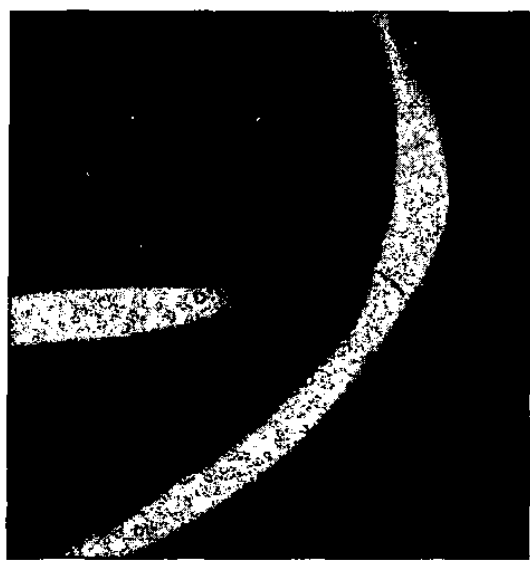

Fig2. Synthecon PGA 63x Sch 810ex

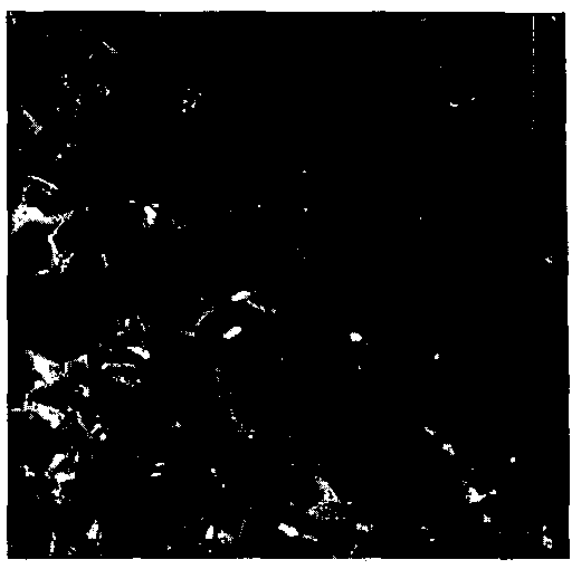

Fig3. PLA 40x 10by10

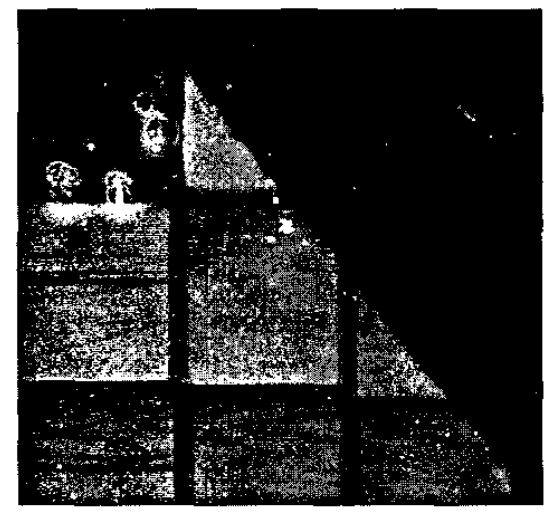

Fig4. chitosan 3by3 40x

\section{Conclusion}

We have demonstrated that multiphoton microscopy can be used to characterize tissue engineering scaffolds constructed from polymers such as PGA. In the future, we will seek to characterize multiphoton signatures of other tissue engineering scaffolds such as collagen and other polymeric materials.

\section{Reference}

[1] P.So, C.Y. Dong, B.R. Masters, et al. "Two-photon excitation fluorescence microscopy. " Annual Review of BiomedicalEengineering, 2,pp. 399-429(2000).

[2]Jyh-Hong Wang, Cheng-Wey Wei,Hwa-Chang Liu,Tai-Hong Yonung "Behavior of MG-63 cells on nylon/chitosan-blended membranes", Biomaterials, accepted (2002)

[3] Y.C. Wang, M.C. Lin, D.M. Wang, H.J. Hsieh, "Fabrication of a novel porous PGA-chitosan hybrid matrix for tissue engineering," Biomaterials, 24, 1047-1057 (2003). (SCI) 